\title{
Analysis of the phase lapse problem in closed interferometers
}

\author{
M. Ţolea ${ }^{\mathrm{a}}$, V. Moldoveanu ${ }^{\mathrm{a}}$, I.V. Dinu $^{\mathrm{a}}$, B. Tanatar ${ }^{\mathrm{b}, *}$ \\ a National Institute of Materials Physics, POB MG-7, 77125 Bucharest-Magurele, Romania \\ ${ }^{\mathrm{b}}$ Department of Physics, Bilkent University, Bilkent, 06800 Ankara, Turkey
}

\section{A R T I C L E I N F O}

\section{Article history:}

Received 18 May 2012

Accepted 12 July 2012

Available online 20 July 2012

Communicated by V.M. Agranovich

\section{Keywords:}

Transmittance phase

Aharonov-Bohm interferometer

Fano parameter

\begin{abstract}
A B S T R A C T
We investigate the connection between the asymmetry of the Fano resonances in a mesoscopic interferometer with an embedded quantum dot and the $\pi$ lapses in the phase of the "bare" dot transmittance. Consecutive Fano resonances with the same (opposite) sign of the Fano parameter imply the presence (absence) of a phase lapse with $\pi$ between the corresponding resonances of the dot. Our results suggest that the famous "phase lapse" problem, first reported by Schuster et al. [R. Schuster, E. Buks, M. Heiblum, D. Mahalu, V. Umansky, H. Shtrikman, Nature 385 (1997) 417], can therefore be experimentally addressed in closed interferometers. It is also proposed that the Fano effect can be used to extract the phase distributions of the eigenfunctions for a mesoscopic 2D shape, via the parity of the resonances. In the presence of electron-electron interaction, one can calculate the phases of the T-matrix elements. The numerical results lead to the same conclusions as for the non-interacting case.
\end{abstract}

(c) 2012 Elsevier B.V. All rights reserved.

\section{Introduction}

The phase coherence lies in the very definition of mesoscopic physics and plays a crucial role in cornerstone interference experiments at nanoscale (Aharonov-Bohm oscillations, mesoscopic Fano effect). Of particular interest is the phase of an electron tunneling through a quantum dot. Unfortunately, such a phase is not directly accessible in the typical transport set-up where the dot is connected to source and drain leads. On the theoretical side the most relevant transport quantities are usually derived as statistical averages of quantum objects (current, occupation numbers) in which the phase information is lost. The way out of these difficulties in phase measurements is to use interference geometries.

In a first attempt to measure the transmittance phase, Yacoby et al. [1] performed transport measurements on a two-lead mesoscopic ring with a QD inserted in one arm, the phase being extracted from a simple two-path interference formula. This system is also called (in a slightly misleading manner) "closed" interferometer, as opposed to the "open" interferometer, which will be described below. The main experimental problem, however, with the "closed" set-up is that the electrons encircle the ring more than once, blurring the single-tunneling phase information. Moreover, if a magnetic field is applied, the resulting AharonovBohm $(A B)$ oscillations have to be symmetric with respect to the field reversal due to Onsager relations and therefore one cannot expect to extract phase information from the shift of the oscillations.

\footnotetext{
* Corresponding author. Tel.: +90 3122901591.

E-mail address: tanatar@fen.bilkent.edu.tr (B. Tanatar).
}

The problem was solved by Schuster et al. [2] who "opened" the interferometer by adding supplementary base zones to absorb the deflected electrons and ideally to ensure just a single interference. In this way, one can measure not only the amplitude of the transmission, but also its phase, which is extracted from the $A B$ conductance oscillations. The principle is simple: if the partial electronic wave traveling through the $\mathrm{QD}$ acquires a supplementary phase, then this phase must be compensated with a change in the magnetic flux, in order to preserve the same interference pattern. Therefore, the supplementary phase introduced by the dot is simply equal to the phase shift of the $A B$ oscillations.

The Friedel sum rule [3] (see also [4-6]) and other simple models, like the 1D double barrier (see Fig. 14 in Ref. [7]) suggest that the measured phase increases with $\pi$ on each QD resonance and remains at a constant value between resonances. Surprisingly, the experiment [2] found instead that between any two consecutive conductance peaks the transmission phase displays a jump (phase lapse) of $\pi$. The transmittance phase measurements in open interferometers was taken a step further by Avinun-Kalish et al. [8] who controlled the QD occupancy via a nearby quantum point contact. The QD was first depleted of electrons and then it is gradually filled. For the first few electrons added to the dot $(N<10)$, the authors reported a non-universality of the phase behavior, which varied on some resonances and between them with $\pi$ or fractions of $\pi$. This regime was called "mesoscopic". In the multi-electron regime, the "universal" phase lapse with $\pi$ emerges, as obtained in all the previous experiments.

The phase lapse problem is considered by some authors to be "the longest standing puzzle in mesoscopic physics" (see, e.g., Ref. [9]) as the experimental results were not entirely reproduced by the various theoretical models proposed so far. 
Many theoretical papers used discrete models for the analysis of the transmittance phase (see, e.g., $[10,11,7,12,13]$ ), especially for the non-interacting case. Among the first papers addressing theoretically the behavior of the phase were Refs. $[10,11]$, where it was shown that phase lapses between two resonances can be associated with a zero of the conductance at the corresponding energy, and this happens between consecutive resonances with the same parity.

The experiments by Avinun-Kalish et al. [8] generated a new direction of theoretical research employing sophisticated manybody techniques to explain the phase behavior in the few-electron regime [14-16]. Other authors addressed the crossover from mesoscopic to universal regime [17-19]. The emergence of the universal phase lapses regime was explained by some authors by a "population switching" inside the quantum dot [20,21]. In Ref. [22], a set-up consisting of a double-dot mesoscopic ring and a nearby charge detector was proposed. It is shown that the "bare dot" phase evolution can be extracted from the second harmonic of the $\mathrm{AB}$ oscillations.

At this point, it is important to stress that even if the open interferometer solves the problem of multiple interferences, it also has a number of important drawbacks from experimental point of view, in comparison with the closed interferometer: a substantially reduced signal, and some uncertainties regarding the influence of the base zones themselves on the measured phase. From the theoretical point of view, the open interferometer is difficult to be described by a simple Hamiltonian, even if some efforts have been made [23].

Bearing in mind the mentioned drawbacks of the open interferometer, our goal in this Letter is to show what phase information can be extracted from closed interferometers. If a variable gate potential is applied on a QD inserted in one arm of an $A B$ interferometer, the result in conductance would be asymmetric Fano resonances, with the general equation $\mathcal{G} \approx(\epsilon+q)^{2} /\left(\epsilon^{2}+1\right)$, $q$ being called the Fano parameter. We prove that the sign of $q$ carries the important information about the existence (or not) of a phase lapse with $\pi$ of the "bare" dot transmittance.

The correspondence we shall prove can be used to interpret already existing experimental results, that used closed interferometers in the context of the Fano effect, but did not discuss phase implications. Some existing experimental data suggest that, even if the phase lapses are indeed present almost between any pair of resonances, they are not universal, as out-of-phase resonances are also present [24,25].

We shall also present some results for the interacting case, which also suggest that the $\pi$ lapse of the phase between resonances can be predicted from the sign of consecutive Fano parameters, similar to the non-interacting case.

The outline of the Letter is as follows: Section 2 contains the analytical and numerical results for the non-interacting case, Section 3 contains the results for the interacting case, while the implications of our results and the conclusions of the Letter are summarized in Section 4.

\section{The phase information embedded in the sign of the Fano parameter: the non-interacting case}

We consider a multi-level quantum dot embedded in a mesoscopic interferometer (see the left sketch in Fig. 1). The system will be described by a lattice model, the QD being of arbitrary shape and coupled to the ring in the sites $i_{\alpha}$ and $i_{\beta}$. For simplicity we consider that the "ring" is quite simple and consists of only two sites $\alpha$ and $\beta$ connected by the hopping constant $\tau_{\alpha \beta}$. Then the Hamiltonian describing the mesoscopic interferometer and the left and right leads coupled to it reads as follows:

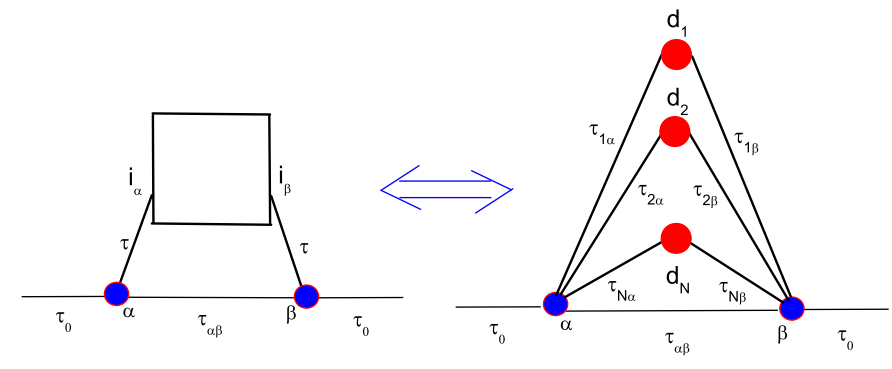

Fig. 1. (Color online.) Coupling a QD to the sites $\alpha$ and $\beta$ is equivalent to individually coupling each QD state to the leads. In the latter case, the couplings are no longer equal and can have different signs, as they are proportional to the value of the eigenfunctions in the site $i_{\alpha}$ or $i_{\beta}$, respectively (see description in text). In order to form an interferometer, the sites $\alpha$ and $\beta$ are inter-connected by the hopping $\tau_{\alpha \beta}$ and connected to the leads by $\tau_{0}$

$H=H_{D}+H_{R}+\sum_{\gamma=\alpha, \beta} H_{L_{\gamma}}+H_{D R}+H_{T}$

where $H_{D}, H_{R}$ describe the QD and the ring, while $H_{L_{\gamma}}, H_{T}$ and $H_{D R}$ represent the leads Hamiltonian and the tunneling Hamiltonians, respectively:

$$
\begin{aligned}
& H_{L_{\gamma}}=\sum_{k_{L_{\gamma}}} \epsilon_{k}\left|k_{L_{\gamma}}\right\rangle\left\langle k_{L_{\gamma}}\right|, \\
& H_{T}=\tau_{0} \sum_{k_{L_{\gamma}=\alpha, \beta}}\left|k_{L_{\gamma}}\right\rangle\langle\gamma|, \\
& H_{R D}=\tau\left(|\alpha\rangle\left\langle i_{\alpha}|+| \beta\right\rangle\left\langle i_{\beta}\right|\right) .
\end{aligned}
$$

Let us first briefly review an important result obtained first in Ref. [10] connecting the phase evolution between consecutive resonances (more precisely the existence or not of a phase lapse with $\pi$ ) with the parity of the respective resonances. Then we shall insert the same QD in an interferometer, and prove that the information on the resonances parity can be extracted from the sign of the Fano parameter, for consecutive Fano lines. We remind here that the parity of a resonance associated to the $n$th state $\Psi_{n}$ of the dot is defined to be the sign of the product $\Psi_{n}\left(i_{\alpha}\right) \Psi_{n}\left(i_{\beta}\right)$. In the absence of Coulomb interaction the conductance is given by the Landauer formula $\left(E_{F}\right.$ is the Fermi level of the leads):

$\mathcal{G}\left(E_{F}\right)=\frac{2 e^{2}}{h} 4 \Gamma^{2}\left|G_{i_{\alpha} i_{\beta}}^{D}\right|^{2}$,

where $G^{D}$ is the effective retarded Green function $G^{D}(E)=(E-$ $\left.H^{D}+\Sigma^{L}\right)^{-1}$, and $\Sigma^{L}=i \Gamma\left(\left|i_{\alpha}\right\rangle\left\langle i_{\alpha}|+| i_{\beta}\right\rangle\left\langle i_{\beta}\right|\right)$ is the lead's selfenergy with $\Gamma=2 \pi \rho_{0} \tau_{0}^{2}, \rho_{0}$ being the density of states in the leads.

Levy Yeyati and Büttiker [10] approximated the Green function as follows ( $E_{n}$ is the energy of the state $\Psi_{n}$ ):

$G_{i_{\alpha} i_{\beta}}^{D}\left(E_{F}\right) \approx \sum_{n} \frac{\Psi_{n}\left(i_{\alpha}\right) \Psi_{n}\left(i_{\beta}\right)}{E_{F}-E_{n}+i \Gamma\left[\Psi_{n}^{2}\left(i_{\alpha}\right)+\Psi_{n}^{2}\left(i_{\beta}\right)\right]}$,

which means that, in the limit of low coupling, the Green function can be written as a sum of resonances, and furthermore, the parameters of interest have simple expressions. Now the question was whether the above formula contains information about the phase evolution between resonances, more precisely about the presence (absence) of a phase lapse. Keeping in mind that the Green function (Eq. (4)) is a complex number, a phase lapse with $\pi$ appears if both the real and the imaginary parts change their sign simultaneously. This means that, in turn, the Green function has a zero at the phase lapse 
position, so one has to look for the zeros of the Green function.

Mathematically, it is easy to show that the Green function in Eq. (4) can vanish between consecutive resonances if and only if their numerators have the same sign. We have to keep in mind that the real parts of the denominators have opposite signs for consecutive resonances (say, $\epsilon_{n}$ and $\epsilon_{n+1}$ ), if the Fermi level is between the resonances (i.e. $\epsilon_{n}<E_{F}<\epsilon_{n+1}$ ). Thus, if one wants the sum of two neighboring resonances to vanish, their nominators must have the same sign [10]. Therefore, the existence (or not) of zeros in transmittance, or, equivalently a phase lapse of $\pi$ between resonances, can be associated to the same (different) parity of consecutive resonances.

It is important to mention that, even if the simple decomposition in Eq. (4) is correct in the limit of low coupling, the fact that the conductance function can be written as a sum of resonances is always true, and also the positions of the Green function's zeros are independent of the coupling strength, as was proven in Ref. [10].

We now turn to the interferometer geometry. In Ref. [26], it was shown that the conductance can be expressed in terms of two effective Green functions for the embedded dot and for the two sites ring:

$\mathcal{G}=\frac{2 e^{2}}{h} 4 \Gamma^{2}\left|\tilde{G}_{\alpha \beta}^{R}+\tau^{2} \sum_{\gamma, \gamma^{\prime}=\alpha, \beta} \tilde{G}_{\alpha \gamma}^{R} \tilde{G}_{i_{\gamma} i_{\gamma^{\prime}}}^{D} \tilde{G}_{\gamma^{\prime} \beta}^{R}\right|^{2}$,

where $\tilde{G}^{D}(E)=\left(E-H^{D}+\Sigma^{D}\right)^{-1}$ and $\Sigma^{D}=-\tau^{2} \sum_{\gamma, \gamma^{\prime}=\alpha, \beta}\left|i_{\gamma}\right\rangle \times$ $\tilde{G}_{\gamma \gamma^{\prime}}^{R}\left\langle i_{\gamma^{\prime}}\right|$. For our simplified model, we can calculate straightforwardly (we assumed the diagonal energies in the ring sites to be zero, and also $\left.E_{F}=0\right)$ :

$\tilde{G}_{\alpha \beta}^{R}=\tilde{G}_{\beta \alpha}^{R}=-\tau_{\alpha \beta} /\left(\tau_{\alpha \beta}^{2}+\Gamma^{2}\right)=: g_{1}$,

$\tilde{G}_{\alpha \alpha}^{R}=\tilde{G}_{\beta \beta}^{R}=-i \Gamma /\left(\tau_{\alpha \beta}^{2}+\Gamma^{2}\right)=: g_{2}$.

Finally, using the notations $\tau_{n \alpha}:=\tau \Psi_{n}\left(i_{\alpha}\right)$ and $\tau_{n \beta}:=\tau \Psi_{n}\left(i_{\beta}\right)$ one obtains

$\Sigma_{n}^{D}=-\sum_{\gamma \gamma^{\prime}=\alpha, \beta} \tau_{n \gamma} \tau_{n \gamma^{\prime}} \tilde{G}_{\gamma \gamma^{\prime}}^{R}$,

for the self-energy corresponding to the dot level $\epsilon_{n}$. Introducing in Eq. (5) the required expressions one arrives at:

$\mathcal{G}=\frac{2 e^{2}}{h} 4 \Gamma^{2}\left|g_{1}+\sum_{n} \frac{\left(\tau_{n \alpha}^{2}+\tau_{n \beta}^{2}\right) g_{1} g_{2}+\tau_{n \alpha} \tau_{n \beta}\left(g_{1}^{2}+g_{2}^{2}\right)}{-E_{n}-\left(\tau_{n \alpha}^{2}+\tau_{n \beta}^{2}\right) g_{2}-2 * \tau_{n \alpha} \tau_{n \beta} g_{1}}\right|^{2}$.

For well separated resonances, the summation symbol $\sum_{n}$ can be placed in front of the square modulus. Then inside the modulus we bring the terms to the same denominator:

$\mathcal{G}=\frac{2 e^{2}}{h} 4 \Gamma^{2} \sum_{n}\left|\frac{-g_{1} E_{n}+\tau_{n \alpha} \tau_{n \beta}\left(g_{2}^{2}-g_{1}^{2}\right)}{-E_{n}-\left(\tau_{n \alpha}^{2}+\tau_{n \beta}^{2}\right) g_{2}-2 * \tau_{n \alpha} \tau_{n \beta} g_{1}}\right|^{2}$.

By replacing $g_{1}$ and $g_{2}$ in Eq. (9) one can convince himself after some manipulation that the conductance can be expressed as a sum of Fano resonances [27]:

$\mathcal{G}=A \sum_{n} \frac{\left(\epsilon_{n}+q_{n}\right)^{2}}{\epsilon_{n}^{2}+1}$

with the following notations:

$q_{n}=\tau_{n \alpha} \tau_{n \beta} \frac{\left(\frac{\tau_{\alpha \beta}}{\Gamma}-\frac{\Gamma}{\tau_{\alpha \beta}}\right)}{\left(\tau_{n \alpha}^{2}+\tau_{n \beta}^{2}\right)}, \quad A=\frac{2 e^{2}}{h} 4 \Gamma^{2} \frac{\tau_{\alpha \beta}^{2}}{\left(\tau_{\alpha \beta}^{2}+\Gamma^{2}\right)^{2}}$, $\epsilon_{n}=\frac{\tau_{\alpha \beta}^{2}+\Gamma^{2}}{\Gamma\left(\tau_{n \alpha}^{2}+\tau_{n \beta}^{2}\right)} E_{n}-2 \frac{\tau_{n \alpha} \tau_{n \beta} \tau_{\alpha \beta}}{\Gamma\left(\tau_{n \alpha}^{2}+\tau_{n \beta}^{2}\right)}$.

The above expression for $q_{n}$ is the main result of our Letter, which shows [keeping in mind that $\tau_{n \alpha} \tau_{n \beta}=\tau^{2} \Psi_{n}\left(i_{\alpha}\right) \Psi_{n}\left(i_{\beta}\right)$ ] that the parity information can be extracted from the sign of the Fano parameter.

The sign of the quantity $\left(\frac{\tau_{\alpha \beta}}{\Gamma}-\frac{\Gamma}{\tau_{\alpha \beta}}\right)$ is not important, because it is independent of the resonance index $n$, and what is important is the relative signs of $q$ (or of the parities) for different resonances. One should mention that, in the presence of a magnetic flux applied on the $A B$ interferometer, the Fano parameter becomes complex [26], however for the purpose of extracting phase information, one does not need a magnetic flux.

At this point we make use of the equivalence parity $\Leftrightarrow$ phase lapse described in the beginning of this section (following Ref. [10]), and now we can finally conclude that the existence (or not) of phase lapses between consecutive resonances can simply be decided by inspecting the (experimentally observable) sign of the Fano parameters for consecutive resonances. This equivalence was suggested in [12], by numerical results, but the analytical proof was missing.

The quantities $\tau_{n \alpha(\beta)}$ (defined in the paragraph above Eq. (7)) are called effective coupling parameters, so one can also say that the Fano parameter $q$ carries information about the relative sign of the effective coupling parameters. In the next section, we will show that this property is maintained also for an interacting toy model.

The analytical results derived in this section are illustrated by a numerical calculation in Fig. 2, for the case of a rectangular quantum dot $(30 \times 20$ sites). In the upper part of Fig. 2, we plot the amplitudes and phases of the first six eigenmodes. Since the eigenfunctions can be considered to be real, by "phase" we mean actually the sign of the wave function. By convention, let us assume that the color red corresponds to the sign "+" and blue to the sign "-".

If our quantum dot is connected to leads (the position of the leads is indicated by the small blue arrows in Fig. 2), the resulting transmittance is plotted in the graph underneath, and also the transmittance phase. If, furthermore, the dot is placed in one arm of an interferometer, one obtains Fano lines - plotted in the lower part of Fig. 2 - with both positive and negative signs of the Fano parameter.

As expected, the numerical results confirm the analytical results presented in this section. The eigenfunctions No. 1, 2, 3, and 6 have the parity "+" and No. 4 and 5 have the parity "-". Evidently, between resonances of the same parity the phase exhibits a $\pi$ lapse, while between resonances of different parity the phase remains constant. On the resonances themselves, the phase evolves with $\pi$, on a gate interval equal to the resonance width.

We notice that the resonances with the same parity generate Fano lines with the same sign for the Fano parameter. It is then clear that by reversing the problem, one can extract the parity and phase behavior from the sign of the Fano parameters of consecutive resonances.

It is interesting to mention that Fano lines can be obtained also if the $\mathrm{QD}$ is side-coupled, rather than inserted in a ring. But in this case, since the coupling sites to the two leads coincide, all resonances have positive parity regardless of the dot shape (the parity is defined as $\Psi\left(i_{\alpha}\right) \Psi\left(i_{\beta}\right)$, and in the case of coupling the dot in a single site, i.e. $\alpha=\beta$, we have $\Psi^{2}\left(i_{\alpha}\right)$ for the parity, so all resonances have even parity). Therefore, the ring geometry is necessary to retrieve non-trivial phase information. Nonetheless, the T-shape coupling reveals itself a specific 

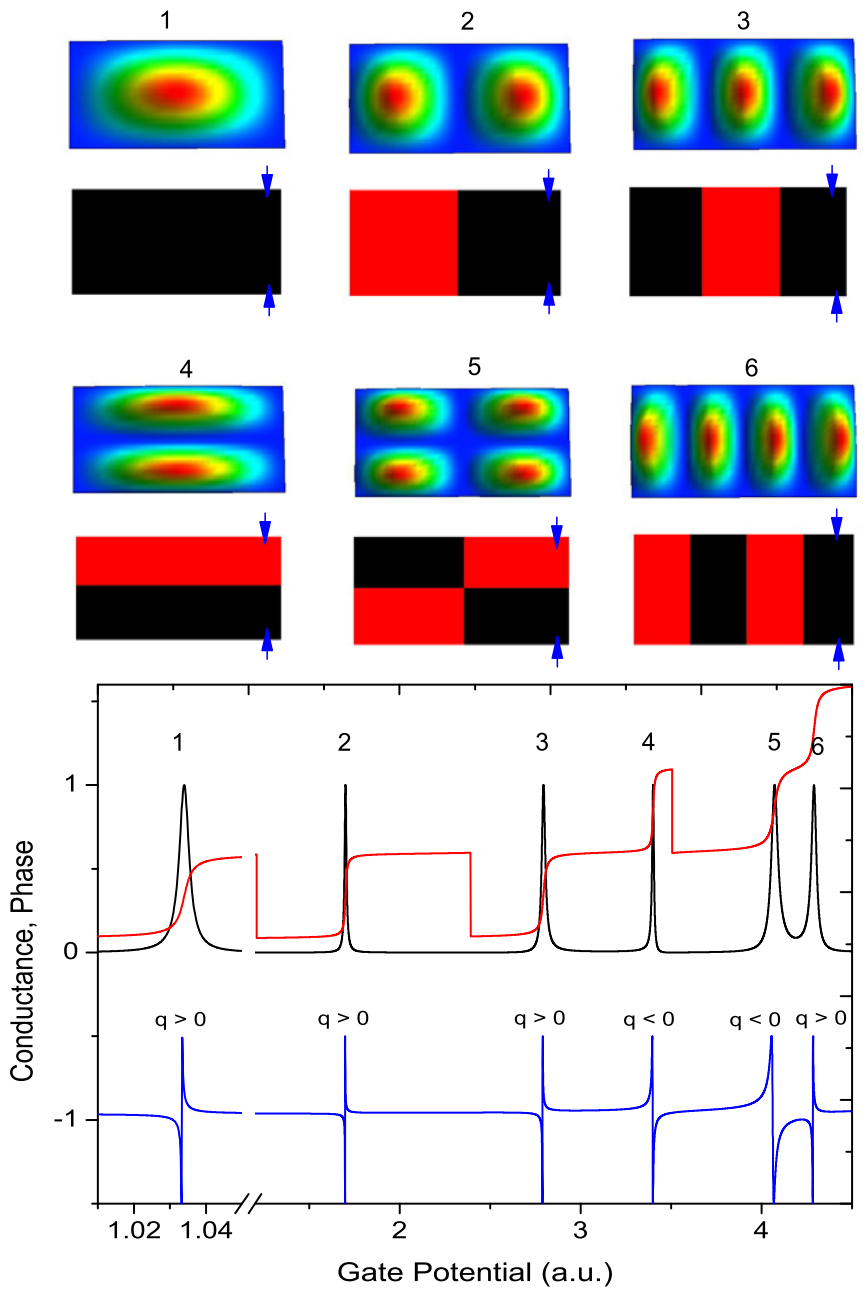

Fig. 2. (Color online.) Up: The first six eigenfunctions of a rectangular QD. The sign distributions of the eigenmodes are plotted below the amplitudes (by convention, we attribute the color black to the sign "+" and the color red to the sign "-"). The small blue arrows indicate the position where the leads will be connected. Down: Transmittance of the quantum dot, amplitude (black, in units of $2 e^{2} / h$ ) and phase (red, in units of $2 \pi$ ) and the corresponding Fano lines (blue, shifted downwards for visibility) which would result by inserting the same dot in an interferometer. Above each Fano resonance we wrote the sign of the Fano parameter. The first resonance is very narrow, so it is plotted with a zoom.

interplay between interference and spin-orbit or Coulomb interactions (see, e.g. the recent papers $[6,28-30]$ and references therein).

\section{The interacting case (a toy model)}

The purpose of this section is to suggest - by the use of a toy model - that the correspondence between the sign of the Fano parameters and the phase lapses (for the "bare" dot) holds also in the presence of interaction.

We shall address the case of a two-level dot, one of them being interacting, with the interaction introduced by the Hubbard term (in the presence of interaction, it is convenient to switch to the second quantization):

$$
\begin{aligned}
H= & \sum_{\sigma, k, \mu=L, R} \epsilon_{k} c_{k_{\mu} \sigma}^{+} c_{k_{\mu} \sigma}+\sum_{\sigma, l=1,2} \epsilon_{l} n_{l \sigma}+U n_{1 \uparrow} n_{1 \downarrow} \\
& +\sum_{\sigma, l=1,2, k, \mu=L, R} \tau_{\mu, l}\left(c_{k_{\mu} \sigma}^{+} d_{l \sigma}+\text { h.c. }\right),
\end{aligned}
$$

with the usual notations (the first and the last terms describe the leads and the leads-dot couplings, the second and third terms are the dot Hamiltonian, with $n_{l \sigma}=d_{l \sigma}^{\dagger} d_{l \sigma}$ ).

The interaction will be addressed in the Hubbard-I approximation, which means the use of the equation of motion technique for the Green functions calculation, and a decoupling performed on the four-operators functions (both the description of this approximation and the current formula have been given in [31]). In particular (see again [31]), we work in the limit $U \rightarrow \infty$, meaning that the on-site Coulomb repulsion is the highest energy scale (much exceeding the single-particle level spacing, etc.). This condition is easily fulfilled in small quantum dots (see, e.g., Ref. [32], where the authors discuss the two-channel Kondo effect and the smaller dot, with the area of $0.04 \mu \mathrm{m}^{2}$, has an estimated Coulomb repulsion $U=1 \mathrm{meV}$, compared to the level spacing of $100 \mu \mathrm{eV}$ ).

If a gate potential is applied on our two-level dot, the conductance will exhibit two resonances, as seen in Fig. 3 (first row). The resonance corresponding to the interacting level is reduced in amplitude, reaching the value $\simeq 2 / 3$, while the resonance corresponding to the non-interacting level reaches 1 . Fig. 3(a) corresponds to the case when both levels are coupled to the leads with positive coupling parameters, while Fig. 3(b) corresponds to the case in which the second level is coupled to one of the leads with a negative coupling parameter. At this point one should mention that in real systems, the occurrence of out-of-phase resonances for the first two electrons added to a system may contradict the results in Ref. [33], which state that the two-electron ground state (at zero magnetic field) has a nodeless orbital part of the wave function. However, our toy model does not refer necessarily to the first two electrons added to the dot, rather it relies on the simplifying assumption that the next two added electrons will be on different orbitals due to the Coulomb repulsion and the coupling of these orbitals to the leads is the same as for the non-interacting case; this section is based on the formalism and approximations described in Ref. [31].

Now, if we place our two-level dot in an arm of an AharonovBohm interferometer, one has in transport the expected Fano resonances, which may have a positive or negative Fano parameter. If the two levels have coupling parameters with the same sign the corresponding Fano lines have the same sign of the Fano parameter $(q>0$ in Fig. 3(c)); if the second level is coupled negatively to one of the leads, its Fano line will have $q<0$, as seen in Fig. 3(d).

In order to continue the comparison with the non-interacting case, one needs at this point to define the relevant phase for the interacting case, which should be extracted from the many-body T-matrix, formally related to the retarded Green function by the definition (the usual notations have been used): $G=G_{0}+G_{0} T G_{0}$ $[34,4]$. We are interested in the propagation from left to right of an electron with $E=E_{F}$ (i.e. $k_{L}=k_{R}=k_{F}$ ). It is straightforward to obtain (assuming also real coupling parameters):

$$
\begin{aligned}
T_{k_{L \sigma} k_{R \sigma}}= & \tau_{L 1} \tau_{R 1} G_{1 \sigma, 1 \sigma}^{r}+\tau_{L 2} \tau_{R 2} G_{2 \sigma, 2 \sigma}^{r} \\
& +\left(\tau_{L 1} \tau_{R 2}+\tau_{L 2} \tau_{R 1}\right) G_{1 \sigma, 2 \sigma}^{r}
\end{aligned}
$$

with the usual notations, $G_{1 \sigma, 1 \sigma}^{r}$ being for instance the retarded Green function $\left\langle\left\langle d_{1 \sigma} \mid d_{1 \sigma}^{\dagger}\right\rangle\right\rangle$, calculated at the Fermi energy, etc. The same approach for the many-body phase problem was proposed in [16]. Our equation (13) coincides with Eq. (7) from Ref. [16], in the limit of zero temperature.

The phase of $T_{k_{L \sigma} k_{R \sigma}}$ is plotted in Fig. 3(a) and (b) (the red lines). Some comments are in order regarding the above formula for the T-matrix element. Evidently, the same formula holds also for the non-interacting case, for a two-level dot, but there are some differences, which shall be discussed in the following. In the absence of interaction, we saw that one can define a transmittance, 

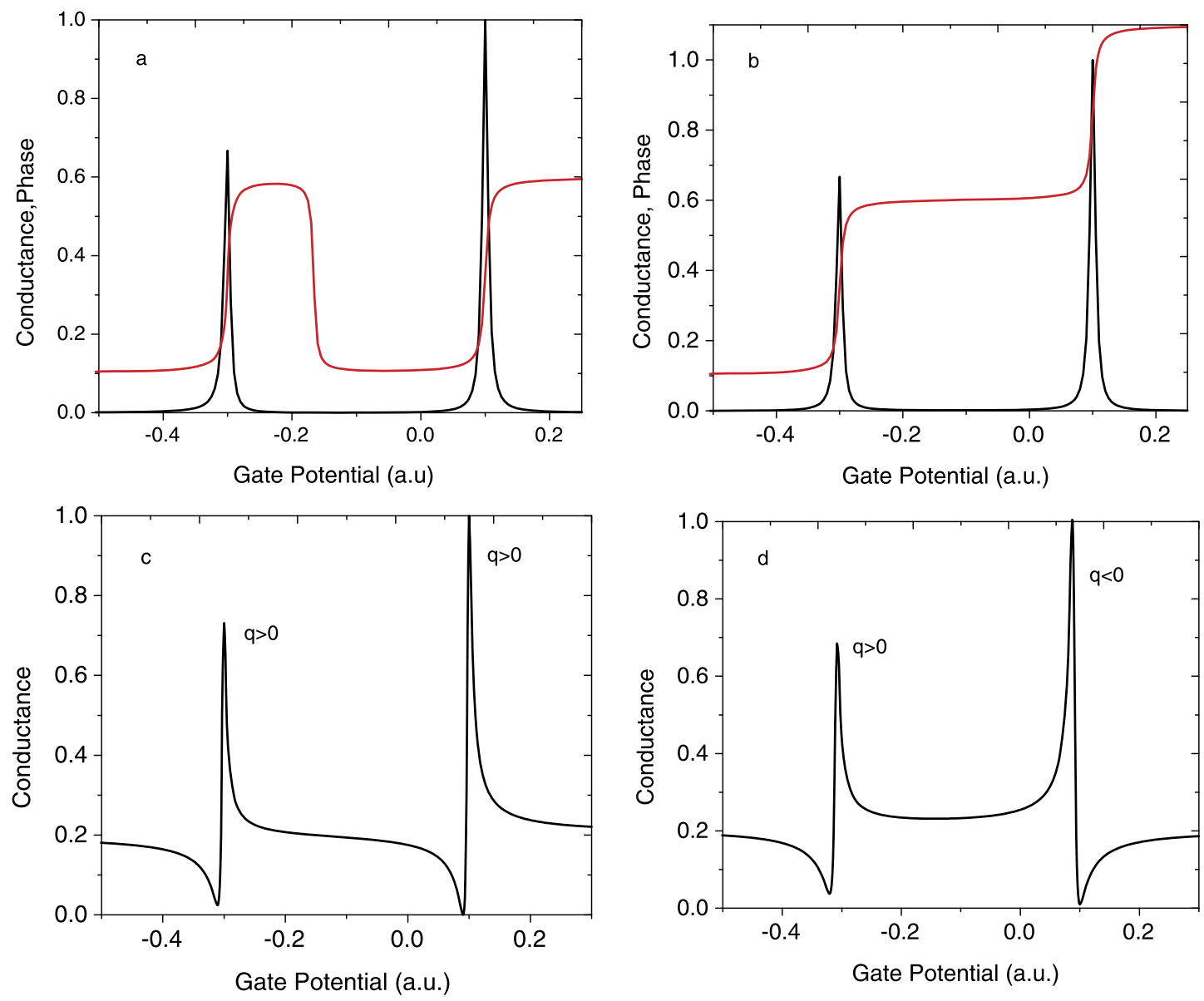

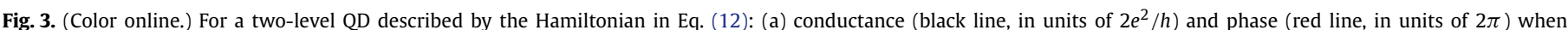

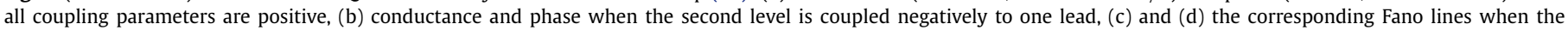
$\mathrm{QD}$ is inserted in a closed interferometer, respectively.

which carries the information for both the conductance (proportional with the modulus of the transmittance) and the phase (equal to the transmittance phase). In the interacting case, the T-matrix element which gives the phase information is no longer correlated with the conductance formula. From Ref. [31], for instance we reproduce the conductance formula for the particular case of asymmetric coupling $\left(\tau_{L 1}=\tau_{R 1}=\tau_{1}\right.$ and $\tau_{L 2}=-\tau_{R 2}=\tau_{2}$, and $\left.\Gamma_{1}=2 \pi \rho \tau_{1}^{2}, \Gamma_{2}=2 \pi \rho \tau_{2}^{2}\right)$ :

$\mathcal{G}=\frac{e}{h} \sum_{\sigma} \operatorname{Im}\left[\left(\Gamma_{1} G_{1 \sigma, 1 \sigma}^{r}+\Gamma_{2} G_{2 \sigma, 2 \sigma}^{r}+2 i \Gamma_{1} \Gamma_{2} G_{1 \sigma, 1 \sigma}^{r} G_{2 \sigma, 2 \sigma}^{a}\right)\right]$.

It may be seen that in the interacting case, there is no longer a connection between the conductance (Eq. (14)) and the T-matrix formula (Eq. (13)). At this point, we present the numerical results for conductance, phase, and the Fano lines which result by inserting the dot in a ring (the ring was simulated by another noninteracting level, whose diagonal energy was kept constant).

The main conclusion is the same as for the non-interacting case: the existence of a phase lapse is related to both resonances having the same coupling parameters. And, similar to the noninteracting case, this corresponds to Fano lines with the same sign of the Fano parameter.

It is interesting to mention, by inspecting Fig. 3(a), that in the presence of interaction, the phase lapses (when present) are resolved in energy, rather than having a sudden "jump" as for the non-interacting case (the same effect can be induced by a finite temperature [35]).

\section{Implications and conclusions}

The usual (and natural) approach if one aims to measure a phase is to use an interference geometry which ensures a single act of interference, and forbids multiple encirclements of the interferometer. In mesoscopic physics, this is done in the socalled "open" interferometers. The open interferometers do however have some drawbacks, and the purpose of this Letter was to show that some important phase information can be extracted also from closed interferometers, in spite of the fact that multiple interferences are in this case allowed and it is generally believed that a correct determination of the phase would be blurred.

If a gate potential is applied on a QD inserted in one arm of a mesoscopic ring (i.e. a closed interferometer), the result in conductance would be an asymmetric Fano resonances. We present detailed calculations directly relating the sign of the Fano parameter - for a dot + ring system - with the behavior of transmission phase between resonances of the "bare" dot. In-phase (outof-phase) neighboring resonances of the dot correspond to Fano line shapes with the same (opposite) sign for the Fano parameter. A lapse with $\pi$ of the phase is present (absent) between in-phase (out-of-phase) resonances.

As mentioned, the important implication is that the famous "phase lapse" problem can be addressed in closed interferometers, simply by inspecting the sign of consecutive Fano parameters. If we apply this interpretation to some existing experiments [24,25], which focused on the Fano effect in closed interferometers (but did not discuss the phase problem) a non-universality of the phase 
lapse aspect can be suggested, as one can notice Fano lines with the same, but also opposite sign of the Fano parameter. This was first suggested in Ref. [12], but the analytical proof was missing, and we provide it in Section 2.

Another result we obtain is that the Fano parameter also carries information about the parity of the resonances (related to the sign on the eigenfunctions in the contact points, see Section 2). This result has an interesting implication, described in the following. Let us imagine that one contact on the QD is fixed while the other can be moved on the QD surface. Both contacts are further connected to a ring and the ring to the leads. A variable gate potential applied on the QD generates Fano lines and the sign of the Fano parameter $q_{n}$ is given by the sign of $\Psi_{n}\left(i_{\alpha}\right) \Psi_{n}\left(i_{\beta}\right)$, for each eigenmode. While, as mentioned, the position of one contact is varied on the QD surface, the Fano parameter will only change sign if the "mobile" contact crossed a nodal line (i.e. reached a zone with opposite sign of the respective eigenmode). Therefore, one can map the sign (phase) distribution of the eigenmodes. The phase mapping of the eigenmodes on a 2D surface was considered to be possible only for isospectral shapes, as proposed by Moon et al. [36] (see also $[37,38])$. In pairs of isospectral shapes, the wave functions can be expressed in terms of each other, which brings a supplementary information, allowing to extract the phase distributions, if the amplitude distributions are known. Our proposed scheme makes use of the Fano effect and it is not necessary for the shape (for which the phase distributions are extracted) to have an isospectral. The drawback however, comes from the fact that present experimental techniques do not allow - to our knowledge - to connect the leads in arbitrary points on a QD surface; rather, the position of the leads is fixed as they are defined by lithography at the same time with the QD, for semiconductor QDs.

The main results provided in this Letter are obtained for the non-interacting model. The case with interaction presents an increased complexity, and an in-depth analysis will be the subject of future work. Such an analysis should be necessary, since the electronic correlations are expected to play an important role in the phase problem (see, e.g. $[8,14-17,20,21]$ ). In the present Letter we presented some results for an interacting toy model, the purpose being again to relate the existence of phase lapses (for the "bare" dot) with the signs of the Fano parameters (of the ring + dot system) for consecutive resonances. Last but not least, we hope that our work will motivate new set of experiments, combining both closed and open interferometers, in order to bring further clarification in the long-debated phase lapse problem.

\section{Acknowledgements}

B.T. acknowledges support from TUBITAK and TUBA. M.T. and V.M. acknowledge the hospitality of Bilkent University where this work was initiated. M.T. and I.V.D. acknowledge financial support from the research grants: PNCDI-II contract TE 90/05.10.2011 and Core program $45 \mathrm{~N} / 2009$.

\section{References}

[1] A. Yacoby, M. Heiblum, D. Mahalu, H. Shtrikman, Phys. Rev. Lett. 74 (1995) 4047.

[2] R. Schuster, E. Buks, M. Heiblum, D. Mahalu, V. Umansky, H. Shtrikman, Nature 385 (1997) 417.

[3] D.C. Langreth, Phys. Rev. 150 (1966) 516.

[4] M. Rontani, Phys. Rev. Lett. 97 (2006) 076801.

[5] V. Vargiamidis, V. Fessatidis, Phys. Lett. A 374 (2010) 4438.

[6] B. Solís, M.L. Ladrón de Guevara, P.A. Orellana, Phys. Lett. A 372 (2008) 4736.

[7] G. Hackenbroich, Phys. Rep. 343 (2001) 463.

[8] M. Avinun-Kalish, M. Heiblum, O. Zarchin, D. Mahalu, V. Umanski, Nature 436 (2005) 529.

[9] Y. Oreg, New J. Phys. 9 (2007) 122.

[10] A. Levy Yeyati, M. Büttiker, Phys. Rev. B 62 (2000) 7309.

[11] A. Silva, Y. Oreg, Y. Gefen, Phys. Rev. B 66 (2002) 195316.

[12] T.-S. Kim, S. Hershfield, Phys. Rev. B 67 (2003) 235330.

[13] M. Tolea, M. Niță, A. Aldea, Physica E 42 (2010) 2231.

[14] M. Rontani, Phys. Rev. B 82 (2010) 045310.

[15] L.O. Baksmaty, C. Yannouleas, U. Landman, Phys. Rev. Lett. 101 (2008) 136803.

[16] C. Karrasch, T. Hecht, A. Weichselbaum, J. von Delft, Y. Oreg, V. Meden, New J. Phys. 9 (2007) 123.

[17] C. Karrasch, T. Hecht, A. Weichselbaum, Y. Oreg, J. von Delft, V. Meden, Phys. Rev. Lett. 98 (2007) 186802.

[18] S.A. Gurvitz, Phys. Rev. B 77 (2008) 201302(R).

[19] R.A. Molina, R.A. Jalabert, D. Weinmann, P. Jacquod, Phys. Rev. Lett. 108 (2012) 076803.

[20] D.I. Golosov, Y. Gefen, Phys. Rev. B 74 (2006) 205316.

[21] M. Goldstein, R. Berkovits, Y. Gefen, H.A. Weidenmüller, Phys. Rev. B 79 (2009) 125307.

[22] V.I. Puller, Y. Meir, Phys. Rev. Lett. 104 (2010) 256801.

[23] A. Aharony, O. Entin-Wohlman, B.I. Halperin, Y. Imry, Phys. Rev. B 66 (2002) 115311.

[24] K. Kobayashi, H. Aikawa, S. Katsumoto, Y. Iye, Phys. Rev. Lett. 88 (2002) 256806.

[25] A. Aharony, O. Entin-Wohlman, T. Otsuka, S. Katsumoto, H. Aikawa, K. Kobayashi, Phys. Rev. B 73 (2006) 195329.

[26] V. Moldoveanu, M. Ţolea, V. Gudmundsson, A. Manolescu, Phys. Rev. B 72 (2005) 085338.

[27] U. Fano, Phys. Rev. 124 (1961) 1866.

[28] V. Moldoveanu, M. Ţolea, B. Tanatar, Phys. Lett. A 376 (2012) 1078.

[29] S. Mukhopadhyay, R. Biswas, C. Sinha, Phys. Lett. A 375 (2011) 2921.

[30] M.L. Vallejo, M.L. Ladrón de Guevara, P.A. Orellana, Phys. Lett. A 374 (2010) 4928.

[31] B.R. Bułka, I.V. Dinu, M. Ţolea, Physica E 40 (2008) 2614.

[32] R.M. Potok, I.G. Rau, H. Shtrikman, Y. Oreg, D. Goldhaber-Gordon, Nature (London) 446 (2007) 167.

[33] D.C. Mattis, The Theory of Magnetism, Harper, New York, 1965.

[34] A.C. Hewson, The Kondo Problem to Heavy Fermions, Cambridge University Press, 1993, see Eq. 1.21, and also Section 5.2.

[35] Y. Oreg, Y. Gefen, Phys. Rev. B 55 (1997) 13726.

[36] C.R. Moon, L.S. Mattos, B.K. Foster, G. Zeltzer, W. Ko, H.C. Manoharan, Science 319 (2008) 782

[37] O. Giraud, K. Thas, Rev. Mod. Phys. 82 (2010) 2213.

[38] M. Ţolea, B. Ostahie, M. Niţă, F. Țolea, A. Aldea, Phys. Rev. E 85 (2012) 036604. 